\title{
Cross-task individual differences in error processing: Neural, electrophysiological, and genetic components
}

\author{
Michael J. Frank \\ University of Arizona, Tucson, Arizona \\ AND \\ Christopher D'Lauro and Tim Curran \\ University of Colorado, Boulder, Colorado
}

\begin{abstract}
The error-related negativity (ERN) and error positivity (Pe) are electrophysiological markers of error processing thought to originate in the medial frontal cortex. Previous studies using probabilistic reinforcement showed that individuals who learn more from negative than from positive feedback (negative learners) had larger ERNs than did positive learners. These findings support the dopamine (DA) reinforcement-learning hypothesis of the ERN and associated computational models. However, it remains unclear (1) to what extent these effects generalize to tasks outside the restricted probabilistic reinforcement-learning domain and (2) whether there is a dopaminergic source of these effects. To address these issues, we tested subjects' reinforcement-learning biases behaviorally and recorded EEG during an unrelated recognition memory experiment. Initial recognition responses were speeded, but the subjects were subsequently allowed to self-correct their responses. We found that negative learners, as assessed via probabilistic learning, had larger ERNs in the recognition memory task, suggestive of a common underlying enhanced error-processing mechanism. Negative learners also had enhanced Pes when selfcorrecting errors than did positive learners. Moreover, the ERN and Pe components contributed independently to negative learning. We also tested for a dopaminergic genetic basis of these ERP components. We analyzed the COMT val/met polymorphism, which has been linked to frontal DA levels. The COMT genotype affected Pe (but not ERN) magnitude; met/met homozygotes showed enhanced Pes to self-corrected errors, as compared with val carriers. These results are consistent with a role for the Pe and frontal monoamines in error awareness.
\end{abstract}

The medial prefrontal cortex is recruited in a wide range of tasks and behaviors, particularly those that involve performance monitoring, including the online detection and correction of errors (Ridderinkhof, Ullsperger, Crone, \& Nieuwenhuis, 2004). Much of the insight gained in this domain has come from the error-related negativity (ERN), an event-related brain potential (ERP) thought to originate in the anterior cingulate, which occurs immediately after subjects make errors in cognitive tasks (Debener et al., 2005; Gehring, Goss, Coles, Meyer, \& Donchin, 1993). The amplitude of the ERN can reflect the degree to which subjects are focused on their mistakes. For instance, subjects show larger ERNs when motivated to improve their accuracy, as compared with those motivated to improve speed (Boksem, Meijman, \& Lorist, 2006). Similarly, individual differences in ERN magnitude have been associated with personality trait measures associated with error processing (Boksem, Tops, Wester, Meijman, \& Lorist, 2006; Pailing \& Segalowitz, 2004).

We recently showed that individuals who were biased to learn more from the negative than from the positive outcomes of their decisions (negative learners) had larger ERNs than did those who showed the reverse behavioral bias ( positive learners; Frank, Woroch, \& Curran, 2005). Negative learners also showed larger conflict-related ERNs when choosing among two stimuli that had both been associated with negative feedback (lose/lose decisions), as compared with two positive options (win/win decisions); positive learners showed the reverse pattern. Furthermore, negative learners had enhanced potentials in response to negative feedback (the feedback-related negativity, FRN) during the learning stage of the task. Together, these data supported the possibility that the ERN and FRN reflect an underlying error correction mechanism, whereby individual differences in this mechanism can cause subjects to learn more from their errors (see also Cohen \& Ranganath, 2007; Hewig et al., 2007). One such mechanism is the transient pause in dopamine (DA) that occurs when outcomes are worse than expected (e.g., Hollerman \& Schultz, 1998). Computationally, these pauses can support one's learning to avoid making maladaptive decisions (Frank, 2005). Support for this model has come from pharmacological studies

M. J. Frank, mfrank@u.arizona.edu 
in which DA levels were altered in healthy subjects and Parkinson's and ADHD patients (Frank \& O'Reilly, 2006; Frank, Santamaria, O'Reilly, \& Willcutt, 2007; Frank, Seeberger, \& O'Reilly, 2004). Moreover, a dopaminergic mechanism is consistent with the reinforcement-learning hypothesis of the ERN, which posits that transient dips in subcortical DA release lead to increased activity in the anterior cingulate, which generates the scalp-recorded ERN (Holroyd \& Coles, 2002).

We could not discern from our previous study whether ERN differences in positive and negative learners were restricted to the particular task investigated or whether they reflected an underlying personality trait that would be apparent across diverse behavioral conditions. It was also not clear to what degree (if any) the dopaminergic system could account for individual differences in learning biases. Here, we addressed these issues in two ways. We assessed positive and negative feedback learning behaviorally and collected ERPs while subjects performed (and made errors in) a completely different recognition memory task. We also collected DNA from the subjects and analyzed the val/met polymorphism within the catechol-O-methyltransferase (COMT) gene. COMT is an enzyme that breaks down DA and norepinephrine and is the primary mechanism by which DA is cleared from the synapse in the prefrontal cortex. The val/met polymorphism has been associated with large individual differences in prefrontal DA, so that met/met individuals have lower COMT enzyme activity and, therefore, higher prefrontal DA than do val carriers (Bilder, Volavka, Lachman, \& Grace, 2004; Egan et al., 2001; Meyer-Lindenberg et al., 2007; Tunbridge, Banner- man, Sharp, \& Harrison, 2004). Furthermore, the COMT enzyme and genotype has been shown to primarily affect prefrontal DA, with little to no effect in the basal ganglia (Gogos et al., 1998; Meyer-Lindenberg et al., 2007). We reasoned that if the ERN is influenced by DA changes in the medial prefrontal cortex (Holroyd \& Coles, 2002), genetic differences in prefrontal DA function would be associated with corresponding differences in the ERN.

We administered a probabilistic selection task designed to test within-subjects biases to learn more from positive or negative feedback (Frank et al., 2004; Frank et al., 2005). Three different stimulus pairs $(\mathrm{AB}, \mathrm{CD}$, and $\mathrm{EF})$ were presented in random order, and the subjects had to learn to choose one of the two stimuli (Figure 1A). Feedback followed the choice, to indicate whether it was correct or incorrect, but this feedback was probabilistic. In AB trials, a choice of Stimulus A led to correct (positive) feedback in $80 \%$ of $\mathrm{AB}$ trials, whereas a $\mathrm{B}$ choice led to incorrect (negative) feedback in these trials (and vice versa for the remaining $20 \%$ of trials). Note that learning to choose A over B could be accomplished by learning either that $\mathrm{A}$ led to positive feedback or that B led to negative feedback (or both). To evaluate the degree to which the subjects learned about the probabilistic outcomes of their decisions (both positive and negative), we subsequently tested them with novel combinations of stimulus pairs involving either an A or a B (each paired with a more neutral stimulus) (Frank et al., 2004). Positive feedback learning was indicated by a reliable choice of Stimulus A in all test pairs in which it was present. Conversely, negative feedback learning was indicated by reliable avoidance of Stimulus B.
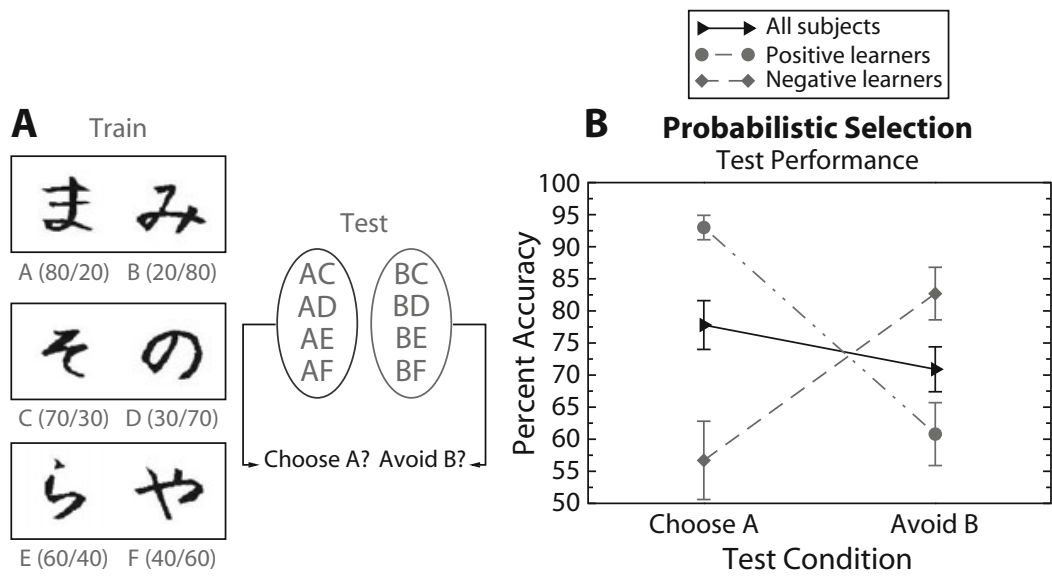

Figure 1. (A) Example stimulus pairs in a probabilistic selection cognitive reinforcement-learning task, in which explicit verbal encoding was minimized by using Japanese Hiragana characters. Each pair was presented separately on different trials. Three different pairs were presented in random order; correct choices were determined probabilistically (percentages of positive/negative feedback are shown in parentheses for each stimulus). A test phase ensued in which Stimuli A and B were re-paired with all other, more neutral stimuli. Positive reinforcement learning was assessed by choose-A performance; aversive learning was assessed by avoid-B performance (Frank, Seeberger, \& O'Reilly, 2004). (B) Behavioral results from the probabilistic selection task. Across all subjects, there was equally good performance for choose-A (positive learning) and avoid-B (negative learning) novel test pairs. Positive learners were better at choosing $\mathrm{A}$, whereas negative learners were better at avoiding B. Error bars reflect the standard error of the mean. 
In our previous study, healthy subjects who were particularly good at avoiding B showed enhanced error- and negative-feedback-related brain activity (Frank et al., 2005). In the present study, relative learning biases were again assessed by a within-subjects accuracy difference in the choose-A and avoid-B conditions. We then recorded ERPs as the subjects performed a different recognition memory task in which they had to respond old or new to items, depending on whether they had previously studied the word on a list. Initial responses were speeded (within $900 \mathrm{msec}$ ), so as to encourage errors. The subjects were then allowed to reverse their responses in order to selfcorrect them if they determined that their initial response was erroneous, following Curran, DeBuse, and Leynes (2007). Thus, dependent measures of the recognition task included accuracy and reaction times (RTs) for the initial response (old/new) and subsequent confirmation or reversal (correction) of this response (see the Method section for details).

We hypothesized that if the ERN reflects a generic error-processing mechanism that is exaggerated in negative learners, these individuals should have larger ERNs when making errors in this different cognitive task. In addition, previous research has shown that unlike the ERN, the error positivity $(\mathrm{Pe})$ is associated with the degree to which subjects are consciously aware of their errors (Nieuwenhuis, Ridderinkhof, Blom, Band, \& Kok, 2001). On the basis of these findings, we hypothesized that larger Pes would be associated with behavioral self-corrections immediately following errors. Finally, we reasoned that negative learners might show an enhanced Pe to selfcorrections if they attended more to their errors than did positive learners.

\section{METHOD}

\section{Subjects}

Fifty-seven University of Colorado students participated in the experiment for course credit or payment. Data from 18 subjects were discarded because (1) the computer crashed during the experiment $(n=8),(2)$ the session time expired before the probability task could be administered $(n=7)$, or (3) there were an insufficient number of trials $(<18)$ in a condition (primarily switch to correct) for ERP analysis $(n=3)$. Finally, in all the positive-/negative-learning analyses, we filtered out 4 subjects who did not perform better than chance $(50 \%)$ on the easiest $\mathrm{AB}$ training pair during the test phase, since their positive/negative learning biases were uninterpretable (Frank et al., 2004; Frank et al., 2005). The remaining 35 subjects were included in the behavioral, genetic, and electrophysiological analyses. Thirtynine subjects were included in the pure genotype/EEG comparisons that did not require comparison of positive and negative learning. Of these 39 subjects, 13 were female, and the mean age was 21 years $( \pm 2.3)$. There were no performance differences in males versus females in either positive or negative learning or in their relative difference $(p s>.5)$.

\section{Probabilistic Selection Task Procedure}

The subjects sit in front of a computer screen in a lighted room and viewed pairs of visual stimuli that were not easily verbalized (Japanese Hiragana characters; see Figure 1). These stimuli were presented in black on a white background, in a 72-point font. They pressed keys on the left or right side of the keyboard, depending on which stimulus they chose to be "correct." Note that the forced choice nature of the task controlled for any differences in overall motor responding. Visual feedback was provided (duration, $1.5 \mathrm{sec}$ ) following each choice (the word "Correct!" printed in blue or "Incorrect" printed in red). If no response was made within $4 \mathrm{sec}$, the words "no response detected" were printed in red.

Three different stimulus pairs $(\mathrm{AB}, \mathrm{CD}$, and $\mathrm{EF})$ were presented in random order. Feedback followed the choice to indicate whether it was correct or incorrect, but this feedback was probabilistic. Choosing Stimulus A led to correct (positive) feedback on $80 \%$ of $\mathrm{AB}$ trials, whereas choosing Stimulus B led to incorrect (negative) feedback on these trials. $\mathrm{CD}$ and EF pairs were less reliable: Stimulus $\mathrm{C}$ was correct on $70 \%$ of $\mathrm{CD}$ trials, whereas $\mathrm{E}$ was correct on $60 \%$ of EF trials. Over the course of training, the subjects learned to choose Stimuli A, C, and E more often than B, D, or F. We enforced a performance criterion (evaluated after each training block of 60 trials) to ensure that all the subjects were at the same performance level before advancing to test. Because of the different probabilistic structure of each stimulus pair, we used a different criterion for each $(65 \% \mathrm{~A}$ in $\mathrm{AB}, 60 \% \mathrm{C}$ in $\mathrm{CD}, 50 \% \mathrm{E}$ in $\mathrm{EF}$ ). (In the $\mathrm{EF}$ pair, Stimulus E was correct $60 \%$ of the time, but this was particularly difficult to learn. We therefore used a $50 \%$ criterion for this pair, simply to ensure that if the subjects happened to "like" Stimulus F at the outset, they nevertheless had to learn that this bias was not going to work consistently.)

The subjects advanced to the test session if all these criteria were met or after six blocks (360 trials) of training. The test session involved presenting the same training pairs, in addition to all the novel combinations of the stimuli, in random sequence. They were instructed (prior to the test phase) to use "gut instinct" if they did not know how to respond to these novel pairs. Each test pair was presented six times, for a maximum of $4-\mathrm{sec}$ duration, and no feedback was provided.

\section{Recognition Memory Task}

Design. The subjects completed six study-test blocks. Two "neutral" blocks were followed by two conservative and two liberal blocks. These additional blocks were utilized to manipulate response bias (i.e., overall tendency to respond old or new), in order to explore the effects of these biases on recognition memory and associated ERP components (Curran et al., 2007). In the conservative blocks, the subjects scored 3 points for correctly responding new and 1 point for correct old responses, whereas in the liberal blocks the payoff bias was reversed. We include these methods here for completeness; this manipulation is not relevant for the present analyses, which focus on ERP responses to erroneous and correct responses regardless of response bias. The neutral blocks were always first; the order of the liberal and conservative blocks (3-6) was counterbalanced in an alternating fashion. Within each test, half of the words were old (studied), and half were new (not studied). EEG data were recorded during the test blocks only.

Stimuli. The stimuli consisted of 480 words broken into six sets of 80 each. The six sets were roughly equated for length $(M=5.29$ letters, $S D=1.06$, range $=4-7)$ and word frequency $(M=16.37$, $S D=19.26$, range $=1-99 ;$ Kučera $\&$ Francis, 1967). These six sets were completely counterbalanced through the three response biases and two old/new conditions. Other words with similar characteristics were used for practice and buffer items.

Procedure. Each session lasted 120-150 min. The subjects completed a consent form, followed by three short practice blocks, the probability task experiment, and the recognition memory experiment. (Note: After Subject 32, the order of tasks was changed to run the recognition memory first, then the probability task at the very end of the session, to reduce fatigue in the longer recognition memory task.)

Test trials included two responses on each trial: a speeded old/new response, followed by a confidence judgment. The subjects situated their hands on a standard keyboard, including the numeric keypad to right, so that their thumbs were positioned over the space (left) and zero (right) keys, whereas the first three fingers of each hand were positioned over the "z," "x," "c" (left keys) and "1," "2," "3" (right 
keys). On each trial, the subjects made a speeded (700-msec deadline) old/new response with their thumbs, followed by a confidence judgment with their fingers. In all blocks, 1 point was deducted for initial studied/nonstudied judgments made after $700 \mathrm{msec}$, regardless of accuracy.

The first and second practice blocks were intended to acquaint the subjects with the key assignments and speeded response procedure. The first practice block consisted of 20 trials on which a 500- to 1,000 -msec fixation sign $(+)$ was followed by either the word "left" or "right." The subjects were instructed to respond with a right or left thumbpress within $700 \mathrm{msec}$ of seeing the corresponding word. After responding, the subjects were given feedback on RT and accuracy. The second practice block included 40 similar trials, except that the words "left" and "right" were replaced with "studied" and "nonstudied." The third practice block included a 10-word study list, followed by a 40 -word test list. Immediately after the third practice block, and every block thereafter, the subjects received feedback about the percentage of first responses made within $700 \mathrm{msec}$, the mean first-response RT, and the mean accuracy of their first responses for that block.

For actual test trials, the subject's first old/new response was followed by a confidence rating. Immediately after the first response, a 6-point confidence interval appeared onscreen (along with the message "too slow" for $500 \mathrm{msec}$ if their initial judgment was after the 700-msec deadline). The terms surely studied, likely studied, maybe studied, maybe nonstudied, likely nonstudied, and surely nonstudied appeared in positions corresponding to the response keys. The subjects made this nonspeeded confidence response using the "z," "x," and "c" keys and " 1, ," 2 ," and " 3 " keys on the number pad with their first three fingers of both hands. The assignment of studied and nonstudied confidence choices to left-/right-hand keys was counterbalanced across subjects, so that they matched the initial studied/nonstudied thumb assignment. For purposes of determining whether initial responses were reversed, we collapsed confidence judgments into two categories: studied (consisting of maybe studied, likely studied, and surely studied) and nonstudied (consisting of maybe nonstudied, likely nonstudied, and surely nonstudied). If this category differed from initial responses, the trial was considered to be a response reversal.

After each test block, a subject's current point total was displayed. After all the subjects had been run, the subject with the highest point total was awarded $\$ 25$.

\section{Electrophysiological Recording and Analysis}

Scalp voltages were collected with a 128-channel Geodesic Sensor Net (Tucker, 1993) connected to an AC-coupled, 128-channel, high-input impedance amplifier (200 M $\Omega$; Net Amps, Electrical Geodesics, Eugene, OR). Amplified analog voltages (0.1 to $100 \mathrm{~Hz}$ bandpass, $-3 \mathrm{~dB}$ ) were digitized at $250 \mathrm{~Hz}$. Individual sensors were adjusted until impedances were less than $50 \mathrm{k} \Omega$. A $40-\mathrm{Hz}$ low-pass filter was applied to the response-locked EEG. Trials were discarded from analyses if more than $20 \%$ of the channels were bad (average amplitude over $100 \mathrm{~V}$ or transit amplitude over $50 \mathrm{~V}$ ). Because blinking was frequent in this long experiment, rather than discarding trials with eye movements, we corrected the EEG from these trials using an ocular artifact correction algorithm (Gratton, Coles, \& Donchin, 1983). Across all the present analyses, the subjects had at least 19 trials per condition. Individual bad channels were replaced on a trial-by-trial basis with a spherical spline algorithm (Srinivasan, Nunez, Tucker, Silberstein, \& Cadusch, 1996). EEG was measured with respect to a vertex reference $(\mathrm{Cz})$, but an average-reference transformation was used to minimize the effects of reference site activity and accurately estimate the scalp topography of the measured electrical fields (Dien, 1998; Picton, Lins, \& Scherg, 1995). The average reference was corrected for the polar average reference effect (Junghöfer, Elbert, Tucker, \& Braun, 1999). Following Yeung, Botvinick, and Cohen (2004) and Frank et al. (2005), response-locked ERPs were computed within epochs starting $800 \mathrm{msec}$ prior to the response and lasting $500 \mathrm{msec}$ after the response and were baseline corrected with respect to the first $100 \mathrm{msec}$ of these epochs.

The means and ranges of trials per condition were as follows: correct $M=260.2, \min =113, \max =352$; incorrect $M=98.9, \min =$ $31, \max =191 ;$ switch-to-correct $M=78.7, \min =18, \max =148$.

ERN. Following other ERN studies using similar recording procedures (Frank et al., 2005; Tucker, Luu, Frishkoff, Quiring, \& Poulsen, 2003), analyses focused on a cluster of seven sensors surrounding Electrode 6 (corresponding to the $\mathrm{Cz}$ location): 5, 6, 7, 12, 13, 107, and 113 . We defined the ERN as the peak-to-peak voltage difference between the first negative peak following the response (mean latency $=32 \mathrm{msec}$ ) and the preceding positive peak (Frank et al., 2005). Peak-to-peak voltage was measured to maintain consistency with prior work (Frank et al., 2005; Holroyd, Praamstra, Plat, \& Coles, 2002; Yeung \& Sanfey, 2004), because the ERN peak was well defined (unlike the Pe), and to minimize possible distortion of the ERN component by the prior positivity (Handy, 2005; Luck, 2005).

Pe. The Pe analysis focused on the Pz electrode (e.g., Falkenstein, Hoormann, Christ, \& Hohnsbein, 2000) and the six surrounding channels $(54,55,61,68,79$, and 80$)$, consistent with reports that the Pe shows a scalp topography similar to that of the P300 (Davies et al., 2001; Leuthold \& Sommer, 1999). The Pe was largest at Sensor 55, the top sensor in our cluster. The average latency of the maximal Pe peak within a 200 - to $500-\mathrm{msec}$ postresponse window was found to be $285.5 \mathrm{msec}$, with a standard deviation of 57.7. The Pe amplitude was calculated by computing the average amplitude within a window that extended one standard deviation before and one standard deviation after the mean latency (228-343 msec postresponse). Unlike the ERN, the Pe peak was less well defined and seemed less influenced by prior components, so we chose a mean amplitude measure (Handy, 2005; Luck, 2005).

P1. As a control for the error-monitoring ERPs, we also analyzed the P1 component from the same Pe electrode cluster. This was the most prominent response-locked component for which we had no reason to predict learning-related differences. We analyzed both the maximum (peak) amplitude of this component and the mean amplitude within a range of one standard deviation around its mean latency.

\section{Genetic}

DNA was collected by use of buccal swabs. The subjects swabbed their cheeks with three cotton swabs, followed by a rinse of the mouth with water, after which all contents were placed in a 50-ml sterile conical tube and stored at $4^{\circ}$ until extraction. Genomic DNA was extracted from buccal cells using a modification of published procedures (Walker et al., 1999). Prior to SNP analyses, the concentration of genomic DNA was adjusted to $20 \mathrm{ng} / \mu \mathrm{l}$. SNP analyses were performed with an ABI PRISM 7500 instrument using TaqMan chemistry. The breakdown of COMT genotypes was 12:16:11 (val/ val:val/met:met/met).

\section{Statistical Analysis}

We performed a standard general linear model regression to test the hypotheses described above, using both continuous measures (choose-A and avoid-B accuracy) and categorical between-subjects variables (met/met vs. val carriers). Where appropriate, we also included repeated measures multivariate analyses to test for interaction effects (e.g., on choose-A vs. avoid-B accuracy, or on Pe to corrected vs. uncorrected errors)

\section{RESULTS}

Across all subjects, there was no difference between choose-A and avoid-B performance [Figure 1B; the slight numerical advantage for choose-A was not significant, $F(1,34)=1.5$, n.s.]. Nevertheless, individual subjects differed in their relative accuracy in these conditions. As in the previous study, we identified two subgroups of sub- 
jects (Frank et al., 2005). Positive learners $(n=18)$ were selected as those subjects who performed better at choosing $\mathrm{A}$ than avoiding $\mathrm{B}$ in novel test pairs, whereas negative learners $(n=13)$ were selected on the basis of better avoid-B performance (Figure 1). Four subjects displayed equally good performance in choose-A and avoid-B test pairs and were not included in either group (but are included in the continuous measure of relative learning biases below). Group comparisons confirmed that positive learners were better than negative learners at choosing A $[F(1,29)=40.9, p<.0001]$, whereas negative learners were better than positive learners at avoiding $\mathrm{B}[F(1,29)=$ $10.3, p=.003]$.

\section{Recognition Memory Performance}

Initial old/new judgments were, on average, $61 \%$ correct. This recognition memory performance did not vary according to positive-/negative-learning bias $[F(1,33)=$ $0.4]$. Across all subjects, $43 \%$ of the errors were subsequently corrected, whereas $6.4 \%$ of the correct choices were inappropriately reversed. This difference was highly significant $[F(1,38)=363.0, p<.0001]$, demonstrating that the subjects successfully utilized their second response opportunity to correct initial inaccurate responses. Behavioral self-corrections did not differ according to relative positive-/negative-learning bias $[F(1,33)=1.0]$. Nevertheless, there was a trend for a negative-learning bias (a relative avoid-B, as compared with choose-A, accuracy) to be associated with increased likelihood of reversing choices that had actually been correct $[F(1,33)=$ 2.9. $p=.096]$. We will return to this issue in the Discussion section.

\section{Error-Related Negativity}

As was expected, the peak-to-peak ERN magnitude following erroneous recognition memory responses was larger than that following correct choices $[-2.41 \mu \mathrm{V}$, as compared with $-2.08 \mu \mathrm{V} ; F(1,33)=12.4, p=.001]$. The ERNs to errors that were subsequently corrected (switch to correct) were largest of all $(-2.74 \mu \mathrm{V})$, significantly larger than those to uncorrected errors $[F(1,33)=6.1, p=$ $.02]$. A general linear model regression revealed that the degree to which the subjects learned more about negative outcomes (as assessed by avoid-B, relative to choose-A, accuracy in the probabilistic task) was significantly associated with larger overall ERN magnitudes in the recognition memory task $[F(1,33)=4.7, p=.038$; see Figure 2]. This relationship was found in terms of a main effect of ERN magnitude on learning biases, which did not interact with error condition [correct choices, errors, or corrected errors; $F(2,66)=0.0]$.

ANOVA group comparisons were consistent with the continuous measures described above. Specifically, negative learners as a group had significantly larger ERN
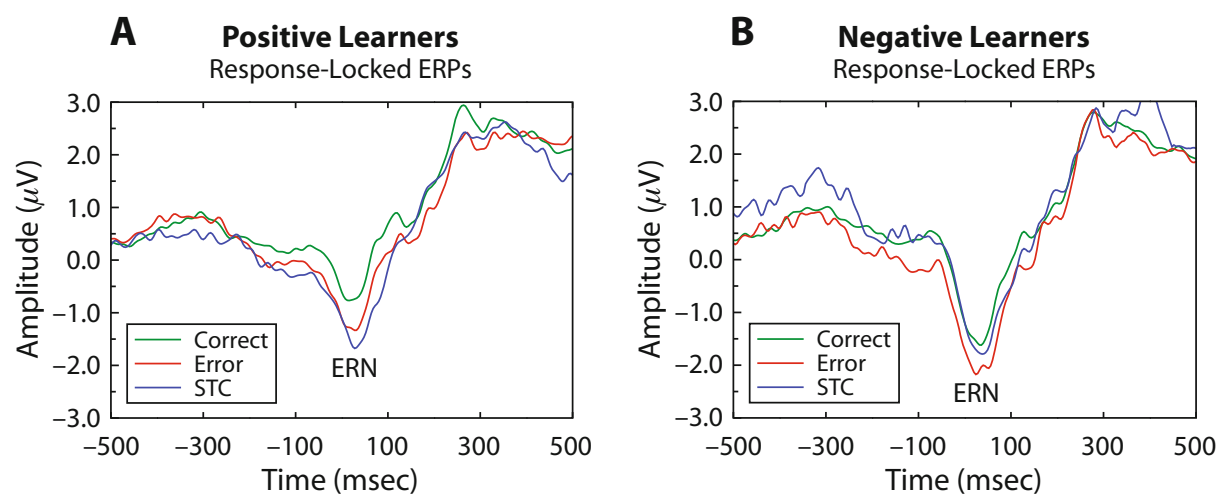

C

D
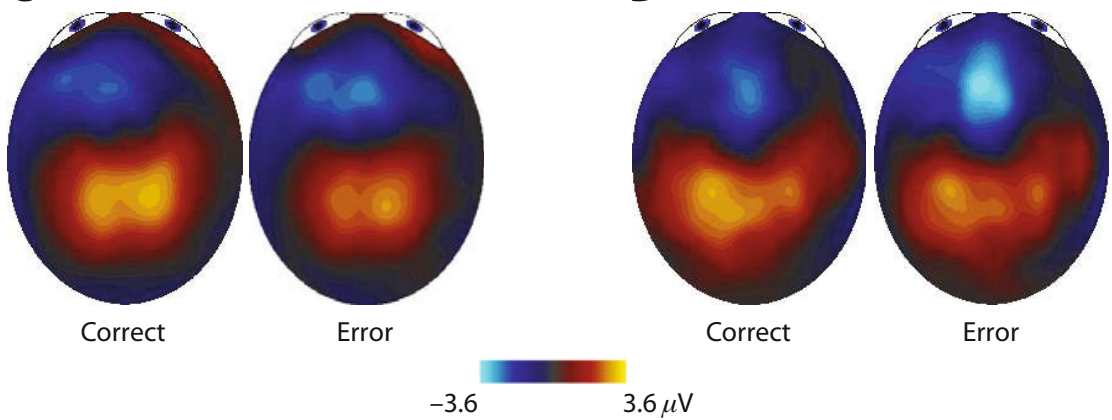

$-3.6$

$$
3.6 \mu \mathrm{V}
$$

Figure 2. Response-locked ERPs during correct and erroneous choices in the recognition memory task. Grand average waveforms are shown separately for positive (A) and negative (B) learners, as determined behaviorally by the probabilistic learning task. Larger error-related negativities (ERNs) were observed in negative learners; this effect did not interact with error condition. Scalp topographies are shown 32 msec postresponse (the peak of the ERN) across correct, uncorrected error, and switch-to-correct (STC) conditions for positive (C) and negative (D) learners. 
amplitudes than did positive learners $[F(1,29)=5.4$, $p=.027$; see Figure 2], with no interaction with error condition [correct choices, errors, or corrected errors; $F(2,58)=0.01]$.

\section{Error Positivity}

As was expected, the Pe varied by error condition $[F(2,66)=3.5, p=.03]$. Planned comparisons revealed that the Pe was larger to errors that were subsequently selfcorrected, as compared with correct choices $[F(1,33)=$ $6.4, p=.017]$, and was also marginally larger than that to uncorrected errors $[F(1,33)=3.9, p=.057]$. There was no difference in Pe magnitude to correct choices and uncorrected errors $[F(1,33)=0.0]$. Overall, these results support the notion that the Pe reflects conscious awareness that an error was made (Nieuwenhuis et al., 2001), which is accompanied by a greater tendency to correct these mistakes when the Pe is large.

Like the ERN, there was a trend for reinforcementlearning biases to affect the Pe, in that relatively better negative learning was associated with overall larger Pe magnitudes $[F(1,33)=3.3, p=.076]$. This effect was reliable only for Pes to self-corrected errors [see Figure 3; $F(1,33)=4.0, p=.05]$. The relatively enhanced Pe to self-corrected errors in negative learners suggests that these subjects paid more attention to their errors after becoming aware of them.

Finally, to provide additional confirmation that our findings reflect group differences in ERPs related specifically to error monitoring, we also analyzed early response-locked potentials in the Pe electrode cluster, which should be unrelated to error processing. Specifically, we analyzed the P1 amplitude between -100 and +70 msec with respect to RT; this was the most prominent response-locked component for which we had no reason to predict learningrelated differences. We analyzed both mean and maximum amplitudes of this $\mathrm{P} 1$ component. In contrast to the ERN and Pe, neither mean nor maximum P1 amplitudes varied in proportion to reinforcement-learning bias (relative positive to negative learning), in any of the conditions (correct, error, or switch to correct; all $p$ s n.s.).

\section{Independent Contributions of ERN and Pe to Negative Learning}

This previously unobserved correlation between the Pe and negative learning raises the question of which electrophysiological marker, the ERN or the Pe, is more relevant to negative-learning biases. Whereas some studies have suggested a similar locus for the two components in the rostral and caudal anterior cingulate (Herrmann, Römmler, Ehlis, Heidrich, \& Fallgatter, 2004; van Veen \& Carter, 2002), others have been more equivocal and have suggested a more posterior locus for the Pe that is more like that of the P300 (Davies, Segalowitz, Dywan, \& Pailing, 2001; Leuthold \& Sommer, 1999; Miltner, Braun, $\&$ Coles, 1997). To address whether these components make independent contributions to negative learning, we regressed reinforcement-learning bias (positive relative to negative learning) against both ERN and Pe in the same general linear model. In this analysis, a negative-learning bias was associated with both significantly more negative ERNs $[F(1,32)=5.8, p=.02]$ and more positive Pes $[F(1,32)=5.1, p=.03]$ for corrected errors. The fact that both results remained significant in this multiple regression suggests that these components make independent contributions and are not redundant.

To further investigate this issue, we regressed $\mathrm{Pe}$ and ERN amplitudes as a function of raw negative learning and raw positive learning as separate variables (i.e., choose-A and avoid-B performance, rather than the relative within-subjects difference in these conditions). Larger Pes were associated with significantly better avoid-B performance [see Figure $4 ; F(1,32)=5.1, p=.03$ ], and no relationship with choose-A $[F(1,32)=0.0]$. Moreover, the relationship between Pe magnitude and better avoid-B performance was specifically observed for Pes for errors that were subsequently corrected $[F(1,32)=7.5, p=$ $.01]$, and not for uncorrected errors $[F(1,32)=1.7$, n.s. $]$. Finally, Pe magnitudes were relatively larger to corrected versus uncorrected errors with increasing avoid-B performance $[F(1,32)=4.2, p=.05]$.

In contrast to the Pe, ERNs in the recognition task did not vary in proportion to avoid-B performance $[F(1,32)=$
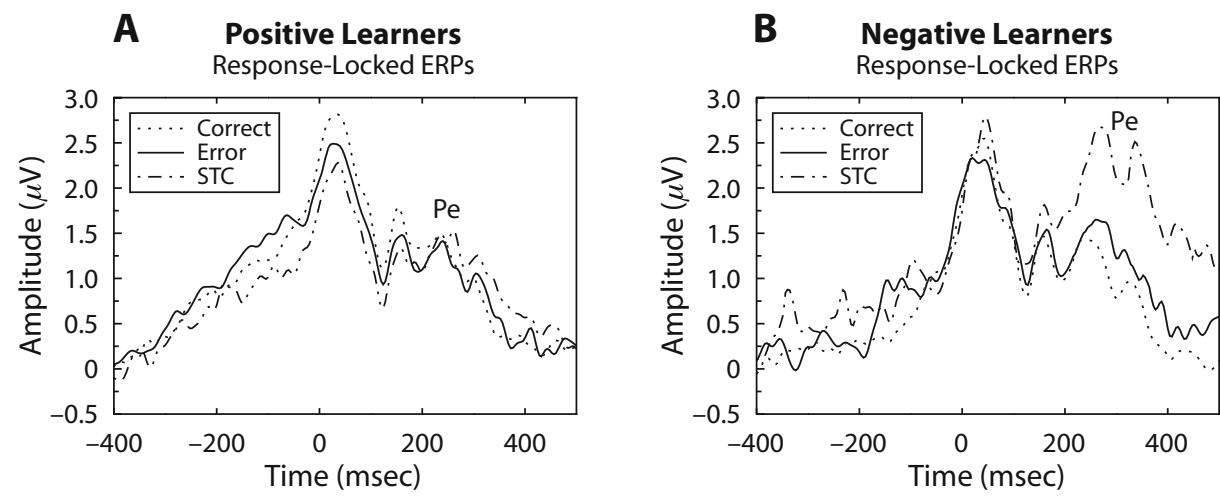

Figure 3. Error positivity (Pe) plotted separately for positive (A) and negative (B) learners. Pes were particularly pronounced in negative learners when correcting errors (STC: switch to correct), with no effect of learning bias on Pes to uncorrected error or correct trials. 


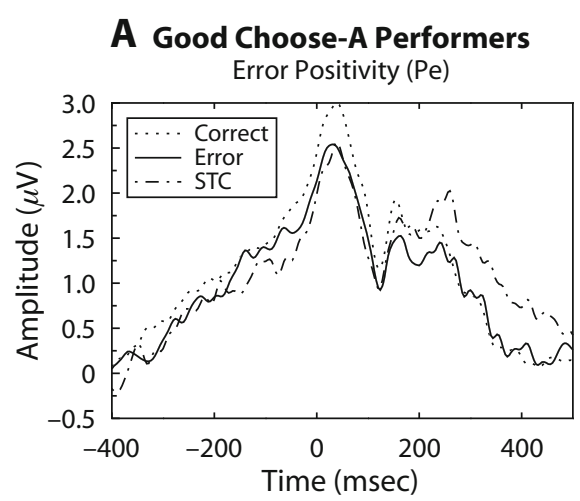

C Good Avoid-B Performers Error Positivity $(\mathrm{Pe})$

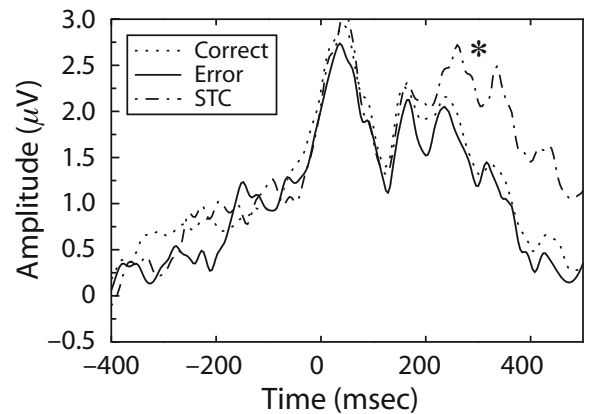

B Poor Choose-A Performers

Error Positivity $(\mathrm{Pe})$

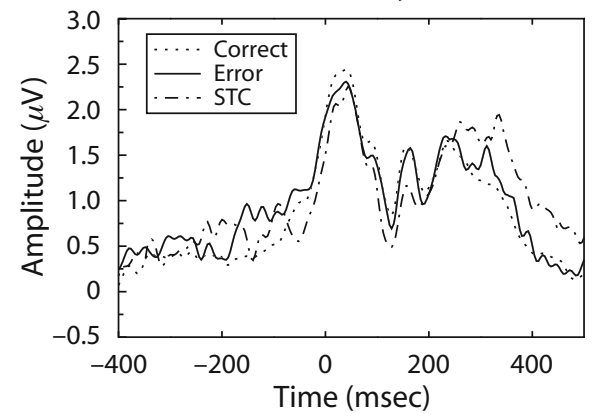

D Poor Avoid-B Performers Error Positivity $(\mathrm{Pe})$

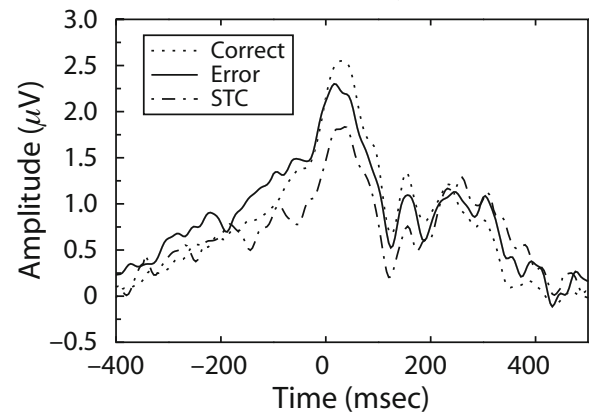

Figure 4. Error positivity (Pe) plotted separately for good $(\mathrm{A}, \mathrm{C})$ and poor $(\mathrm{B}, \mathrm{D})$ choose-A and avoid-B performers, indicative of raw positive and negative learning, respectively. There was no effect of raw positive learning on Pe magnitude; Pes to corrected errors were enhanced only in good raw negative learners, relative to poor raw negative learners $\left({ }^{*} p<.05\right.$ for switch to correct [STC], relative to errors, in good avoid-B performers and for raw STC in good, relative to poor, avoid-B performers). ERP effects were computed using continuous measures of choose-A and avoid-B performance; plots are shown here for display purposes in good and poor performers as defined by median split.

0.7]. Rather, larger ERNs were associated with significantly worse positive choose-A performance [see Figure $5 ; F(1,32)=5.5, p=.025]$. This ERN association with choose-A performance was particularly evident for ERNs in response to actually correct memory judgments $[F(1,33)=7.1, p=.01$; see the asterisk in the figure $]$. In other words, subjects who learned better from correct choices in the probabilistic learning task had smaller ERNs when they were correct in the recognition memory task. Overall, these results are consistent with the notion that positive learners are less likely to think that they have made an error (and may be more confident that they are correct), leading to smaller ERNs.

Thus, these data suggest that a large ERN magnitude is associated with an inability to focus on positive choices, whereas larger Pes are more directly associated with negative learning. However, the ERN results conflict somewhat with those observed in our previous study, in which larger ERNs recorded during the probabilistic learning task correlated directly with raw negative (avoid-B), but not positive (choose-A), learning (Frank et al., 2005). Thus, we remain somewhat cautious about the interpretation of these separable components and emphasize that within-subjects relative-learning biases are most easily interpreted, since multiple factors can affect overall performance. Indeed, using the relative-learning bias measure, we found in both studies that better negative than positive learning was associated with larger ERNs, whether recorded during the probabilistic task or the recognition memory task. The present findings extend those results, showing that the Pe is also associated with a negative-learning bias.

\section{Genetic Components}

Does prefrontal DA affect ERN or Pe magnitude? As was mentioned above, a prominent theory posits that the ERN is caused by transient dips in DA that occur during error processing, which disinhibit neurons in the anterior cingulate (Holroyd \& Coles, 2002). However, it is equally plausible that any relationship between DA and the ERN is in reverse (Frank et al., 2005). Specifically, the anterior cingulate cortex might itself detect cognitive errors via other nondopaminergic mechanisms and then, via top-down projections, induce a transient DA dip subcortically. This dip would then drive no-go learning in the basal ganglia to avoid making maladaptive choices in the future (e.g., Brown, Bullock, \& Grossberg, 2004; Frank, 2005; Frank et al., 2004). Our genetic data provide us with one way of testing these accounts. The val/met polymorphism of the COMT gene has been reliably associated with individual differences in prefrontal DA levels, so that met/met 

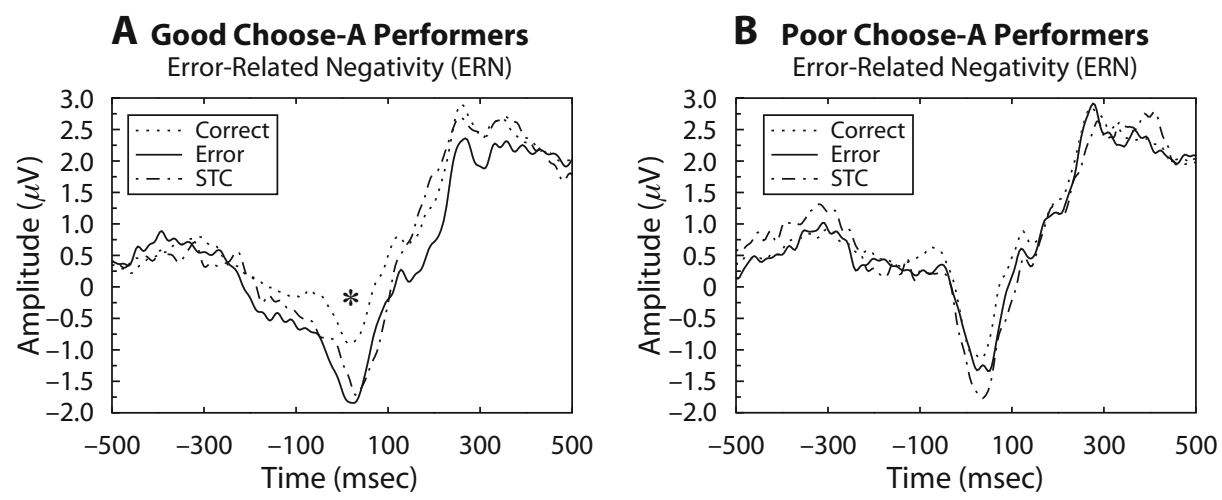

C Good Avoid-B Performers Error-Related Negativity (ERN)
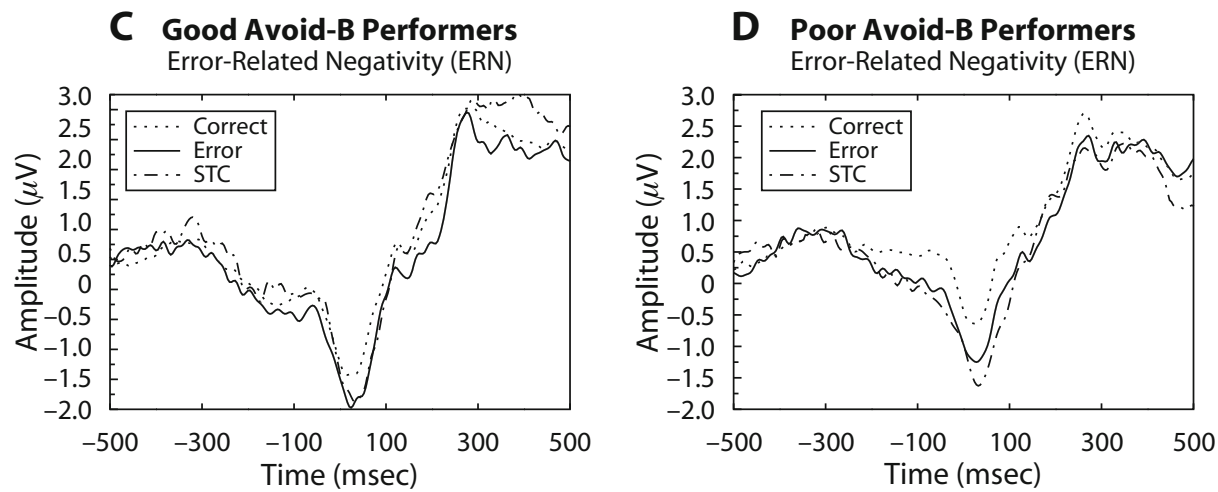

Figure 5. Error-related negativity (ERN) plotted separately for good $(A, C)$ and poor (B, D) choose- $A$ and avoid-B performers, indicative of raw positive and negative learning, respectively. Reduced ERNs were observed in good raw positive learners, especially during correct choices $\left({ }^{*} p<\right.$ .05 , as compared with poor raw positive learners). ERP effects were computed using continuous measures of choose-A and avoid-B performance; plots are shown here for display purposes in good and poor performers as defined by median split. ERN associations with raw avoid-B performance were not significant. STC, switch to correct.

homozygotes have higher levels of prefrontal DA than do val carriers (Bilder et al., 2004; Egan et al., 2001). Holroyd and Coles's account predicts that changes in frontal DA drive the ERN and, therefore, that a genetic polymorphism substantially altering frontal DA levels would measurably affect ERN amplitude. For example, higher tonic DA levels may lead to greater differences resulting from DA dips and, therefore, larger ERNs. In contrast, the alternative hypothesis predicts no such relationship between the COMT and the ERN.

Of the 39 subjects, 11 were homozygous with the met/ met genotype (see the Method section), associated with higher prefrontal DA due to low activity of the COMT enzyme. We compared met/met homozygous with val carriers in both behavioral and electrophysiological measures.

Behaviorally, there was no effect of COMT on positive-/ negative-learning bias $[F(1,33)=0.7]$ or on raw positive or negative learning (both $p \mathrm{~s}>.2$ ). This null effect of COMT on learning biases supports our modeling hypothesis and other empirical data showing that these learning biases result from changes in striatal, rather than prefrontal, DA (Frank, 2005; Frank \& O'Reilly, 2006; Frank et al., 2004). There also was no difference between met/ met and val carriers in initial response accuracies in the recognition memory task $[F(1,37)=0.5]$.
Moreover, there was no effect of COMT genotype on ERN magnitude $[F(1,37)=1.0]$, nor a genotype $\times$ error condition interaction [correct choices, errors, or corrected errors; $F(2,74)=1.4$, n.s.]. (Similar null results held when comparing val/val subjects with val/met and met/met ones). Of course, we cannot reject the Holroyd and Coles (2002) hypothesis due to a null effect of a single gene, even though the COMT gene controls the primary enzyme for regulating frontal DA. Nevertheless, this problem would be somewhat mitigated if we found an effect of COMT on other ERP measures such as the Pe, which would suggest that we did not lack sufficient power to detect such differences.

Indeed, COMT genotype affected Pe magnitudes. There was a trend for a main effect of COMT genotype on Pe $[F(1,37)=3.0, p=.09]$, so that met/met subjects had larger Pes than did val carriers (see Figure 6). Importantly, the interaction between COMT genotype and error condition was significant $[F(2,74)=3.5, p=.036]$. Met $/ \mathrm{met}$ subjects had larger Pes during corrected errors (STC in Figure 6) than did val carriers $[F(1,37)=4.7, p=.036]$. No such relationship was observed for uncorrected errors [Error in the figure; $F(1,37)=0.5]$. Post hoc interaction comparisons revealed that met/met subjects had relatively larger Pes for corrected than for uncorrected errors, as com- 
pared with val carriers $[F(1,37)=5.2, p=.028]$. These effects were found despite no difference among COMT genotypes in the percentage of behavioral self-corrections $[F(1,37)=0.4]$, regardless of correct or erroneous initial responses [interaction between self-correction and error condition, $F(1,37)=0.7]$. Thus, this COMT effect on Pe may reflect enhanced attentional modulation to errors when subjects become aware of them, without affecting the likelihood of behavioral self-correction.

Another possibility is that COMT effects on Pe magnitude reflect differences in motor preparation to the second (corrected) response. This motor account is unlikely, however, since RTs to the second response did not differ across COMT genotypes for either corrected or uncorrected errors $(p s>.4)$. Nevertheless, a post hoc comparison showed a trend for met/met subjects to have relatively faster responses to corrected than to uncorrected errors, when compared with val carriers $[F(1,37)=3.51, p=$ .07]. A similar interaction was found for negative learning, so that better raw avoid-B performance was associated with relatively shorter RTs to corrected than to uncorrected errors $[F(1,33)=6.40, p=.016]$, with no effects on simple RTs ( $p$ s $>.4)$. Thus, we cannot completely discount the possibility that Pe effects reflect differences in motor preparation when responses are reversed. We simply note that enhanced attention to errors would be expected to be associated with more immediate motor preparation so as to correct those errors. Furthermore, the Pe cannot reflect motor execution itself, since it occurred, on average, $286 \mathrm{msec}$ after the initial response, whereas RTs to corrected and uncorrected errors were, on average, 788 and $741 \mathrm{msec}$, respectively, following this response.

The findings above show that the Pe for self-corrected errors is larger in met/met than in val carriers, in negative than in positive learners, and in good than in poor raw negative learners (Figures 3, 4, and 6). However, there was no association between COMT genotype and negative learning. This suggests that COMT and negative learning contribute differential components to Pe magnitudes. When we included both COMT and avoid-B performance in the regression, only avoid-B performance reliably predicted Pe magnitudes to corrected errors $[F(1,32)=$ $6.6, p=.015]$, whereas the COMT effect was no longer significant $[F(1,32)=0.9]$. This suggests that negative learning (which likely has multiple neural and genetic components) contributes more robustly to Pe magnitude, as compared with the COMT genotype.

\section{DISCUSSION}

These findings demonstrate the usefulness of combined behavioral, genetic, and electrophysiological techniques for understanding the neural mechanisms of individual differences in learning and decision making. We found
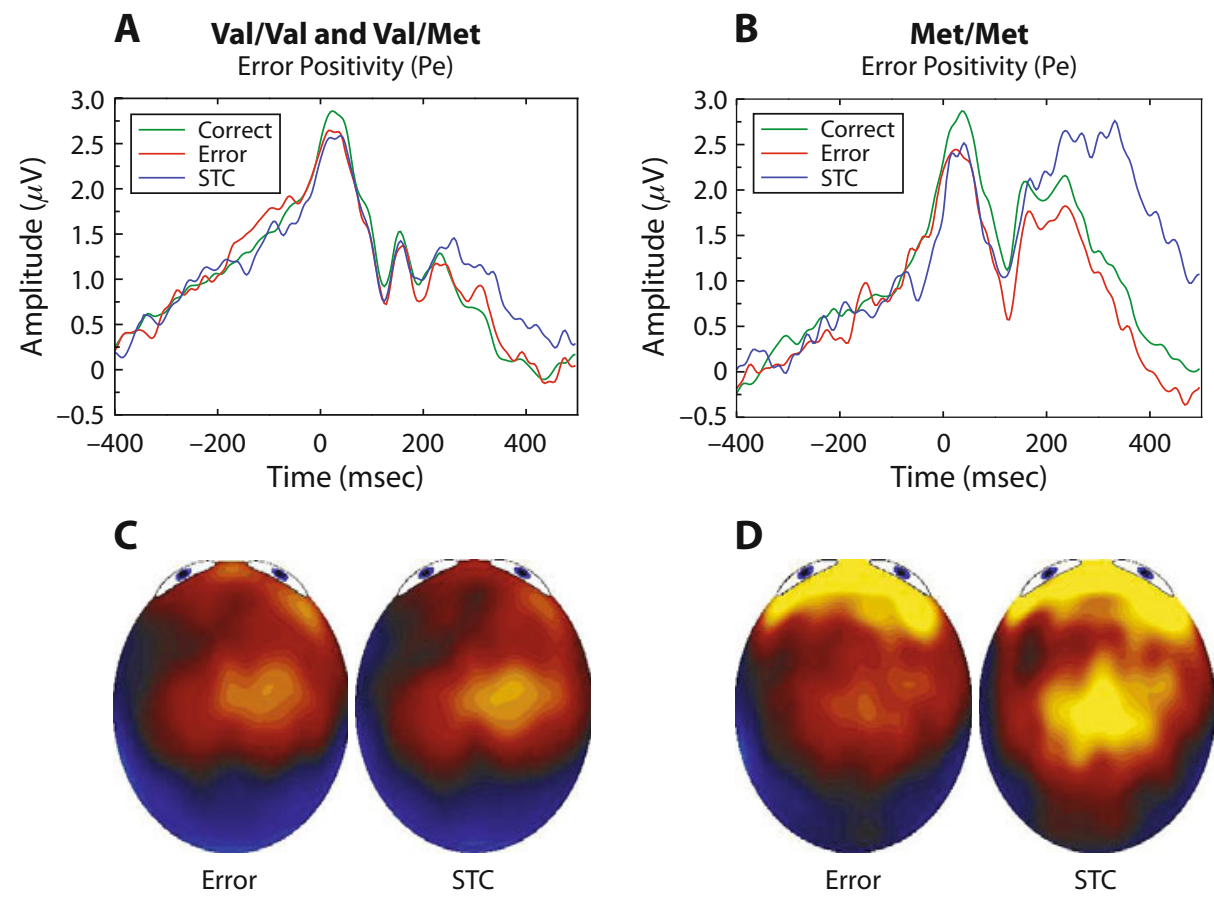

D
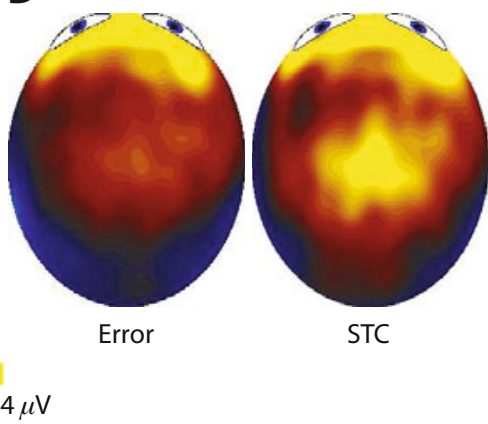

$-4$

Figure 6. Error positivity (Pe) plotted as a function of COMT val/met genotype. (A) Val carriers. (B) Met/met subjects. Pes were particularly pronounced in met/met individuals when correcting errors (STC: switch to correct), with no effect on uncorrected error or correct trials. Corresponding scalp topographies are shown for the different conditions in val carriers $(C)$, and met/met subjects (D), 228-344 msec postresponse (mean \pm one standard deviation of Pe latency; see the Method section). 
that individuals who were better at learning from the negative versus positive outcomes of their decisions had both larger ERNs and larger Pes than did those learning more from positive outcomes. This was true even though the behavioral and temporal context under which ERPs were recorded (the recognition memory experiment) was different from that used to assess positive and negative learning (the probabilistic selection task). Moreover, the ERN and the Pe appear to contribute independently to reinforcement learning biases. Whereas raw negative learning was primarily associated with enhanced Pes, raw positive learning was associated with reduced ERNs. Finally, we showed that a genetic marker of frontal DA, although not associated with learning bias, was predictive of Pe, but not ERN, magnitude. In the following discussion, we will address the significance and interpretation of these collective findings.

During recognition memory judgments, negative learners had larger ERNs than did positive learners. This ERN effect was observed regardless of whether the subjects were actually making errors; negative learners had larger ERNs even to correct responses. It is possible that negative learners are more likely to perceive themselves as having made an error even when they are correct and may, therefore, be less confident in their memory judgments. Supporting this conclusion, there was a trend for a negative-learning bias to be associated with greater likelihood of reversing choices that had initially been correct. Future studies are necessary to further test this accountfor example, by asking subjects to evaluate how often they believed that they had responded correctly. Importantly, objective measures show no difference in actual behavioral performance between positive and negative learners, either in the probabilistic learning (in terms of overall performance accuracy) or the recognition memory task.

The Pe was largest for errors that were subsequently corrected. This finding is consistent with data showing that the Pe is associated with error awareness (Nieuwenhuis et al., 2001). Moreover, our observation that this enhanced Pe to corrected errors was observed primarily in negative learners is novel. The present task paradigm required speeded responses but allowed subsequent reversal of these responses; this aspect may have enabled us to observe this ERP difference among positive and negative learners that was not observed in our prior ERP study focusing on the ERN (Frank et al., 2005). Furthermore, in the present study, the Pe appears to be more associated with negative learning per se, since only the Pe, and not the ERN, reliably predicted raw negative (avoid-B) performance. In addition, the enhanced Pe to corrected errors was observed despite no difference between positive and negative learners in the percentage of errors that were subsequently corrected. This suggests that both positive and negative learners are able to detect that they have made an error in recognition memory judgments and are equally likely to subsequently correct that error but that negative learners may simply attend more to the fact that they erred, leading to a larger Pe.

Further insight into the Pe effect comes from our finding that it was significantly associated with the COMT genotype. Homozygotes for the met allele, who have the highest level of extracellular frontal DA (Bilder et al., 2004; Egan et al., 2001), had larger Pes to corrected errors than did val carriers. Again, met/met subjects did not actually correct their errors more frequently than did val carriers. The COMT contribution to Pe appears to be different from that of negative learning described above: $\mathrm{met} / \mathrm{met}$ individuals did not show a negative-learning bias and were not better at raw negative learning than were val carriers, yet showed larger Pes.

This lack of COMT association with reinforcementlearning biases in the probabilistic task is consistent with computational models and experimental data suggesting that learning biases in this task stem from differences in basal ganglia, and not prefrontal, DA (Frank, 2005; Frank \& O'Reilly, 2006; Frank et al., 2007; Frank et al., 2004). The COMT enzyme and genotype has been shown to primarily affect prefrontal DA, with little to no effect in the basal ganglia (Gogos et al., 1998; Meyer-Lindenberg et al., 2007). In contrast, we have found that a polymorphism in the gene for the DA D2 receptor, which is heavily concentrated in the striatum, as compared with other brain regions, was significantly predictive of negative (avoid-B) learning (Frank, Moustafa, Haughey, Curran, \& Hutchison, 2007). Other indirect evidence that learning biases are not attributable to frontal processes comes from a study in which subjects were administered the drug midazolam, which inactivates the prefrontal (and hippocampal) system. Unlike drugs that alter basal ganglia DA, this pharmacological agent did not affect reinforcement-learning bias or performance in either training or test phases of the probabilistic task, despite having other explicit memory effects (Frank, O'Reilly, \& Curran, 2006).

It is possible that the observed COMT Pe effect reflects a noradrenergic, rather than, or in addition to, a dopaminergic mechanism. The COMT enzyme metabolizes both DA and norepinephrine, so met/met individuals should have not only elevated frontal DA, as is commonly assumed, but also elevated norepinephrine (but see Tunbridge, Harrison, \& Weinberger, 2006, for an argument for why COMT effects are selective to DA). There are two reasons for considering a norepinephrine mechanism for COMT Pe effects. First, several reports have suggested that the Pe is simply a P300 response to salient errors (Davies et al., 2001; Leuthold \& Sommer, 1999; Miltner et al., 1997). Second, a recent compelling review linked the P300 component to phasic norepinephrine release (Nieuwenhuis, Aston-Jones, \& Cohen, 2005). Consistent with this overall interpretation, another recent study showed a COMT effect on P300, where met/met subjects had shorter P300 latencies than did val carriers (Tsai et al., 2003). Finally, the P300, norepinephrine, and COMT have all been associated with novelty seeking (Hansenne, 1999; Kim, Cho, Kang, Hwang, \& Kwon, 2002; Ray, Hansen, \& Waters, 2006; Reuter \& Hennig, 2005; Sara, Dyon-Laurent, \& Hervé, 1995; Tsai, Hong, Yu, \& Chen, 2004). Clearly, future studies are necessary to determine whether COMT effects on Pe are primarily noradrenergic, dopaminergic, or both.

We did not observe an effect of COMT on ERN magnitude. This appears to conflict with the hypothesis that 
the ERN is caused by transient pauses in DA release in the frontal cortex (Holroyd \& Coles, 2002). One would expect that if changes in frontal DA elicit the ERN, a functional polymorphism affecting expression of the primary enzyme controlling frontal dopamine efflux would, in turn, affect the ERN magnitude. Still, larger sample sizes and other behavioral tasks are necessary to confirm a lack of effect. Despite these limitations, as was mentioned above, it is possible that any relationship between DA and the ERN is in reverse. The ERN may reflect a cognitive error detection mechanism in the anterior cingulate that does not itself depend on DA but, instead, causes DA dips subcortically in order to modify learning (Frank et al., 2005). This hypothesis is consistent with the existence of cingulate projections to striosome cells in the striatum, which, in turn, send inhibitory projections to midbrain DA cells (Eblen \& Graybiel, 1995). Finally, it is possible that both mechanisms work in tandem: The subcortical DA system could initially train the medial prefrontal cortex to recognize errors, so that later in training, the medial prefrontal cortex might itself drive DA dips for maladaptive decisions. The latter hypothesis would be consistent with evidence showing that the cingulate learns to predict error likelihood, presumably in advance of any DA dip (Brown \& Braver, 2005).

In conclusion, we have shown that individual differences in reinforcement learning can account for some of the variance in ERP components of error processing, including both the ERN and the Pe. Some of the differences in Pe magnitude could also be attributed to the COMT gene associated with prefrontal DA and norepinephrine function. Nevertheless, further experimental and theoretical work is necessary to unravel the complex relationship between neurotransmitters, ERPs, and state and trait differences in healthy subjects.

\section{AUTHOR NOTE}

This research was supported by NIMH Grant MH64812, NIDA Grant DA022630, the James S. McDonnell Foundation, and the Temporal Dynamics of Learning Center (NSF Science of Learning Center SBE-0542013). Any opinions, findings, and conclusions or recommendations expressed in this material are those of the author(s) and do not necessarily reflect the views of the TDLC or the National Science Foundation. We thank Casey DeBuse, Kristin Becker, Alexis Billings, Chris Bird, Sarah Jirkovsky, Vicky Lai, Maha Sidhom, Brion Woroch, and Brent Young for help with behavioral and electrophysiological data collection. We also thank Heather Haughey, Breanna Miller, and Kent Hutchison for help and resources for DNA extraction and genotyping. Correspondence concerning this article should be addressed to M. J. Frank, Department of Psychology and Program in Neuroscience, University of Arizona, Tucson, AZ 85721 (e-mail: mfrank@u.arizona.edu).

\section{REFERENCES}

Bilder, R. M., Volavka, J., Lachman, H. M., \& Grace, A. A. (2004) The catechol-O-methyltransferase polymorphism: Relations to the tonic-phasic dopamine hypothesis and neuropsychiatric phenotypes. Neuropsychopharmacology, 29, 1943-1961.

Boksem, M. A. S., Meijman, T. F., \& Lorist, M. M. (2006). Mental fatigue, motivation and action monitoring. Biological Psychology, 72, 123-132.

Boksem, M. A. S., Tops, M., Wester, A. E., Meijman, T. F., \& Lorist, M. M. (2006). Error-related ERP components and individual differences in punishment and reward sensitivity. Brain Research, 1101, 92-101.
Brown, J. W., \& Braver, T. S. (2005). Learned predictions of error likelihood in the anterior cingulate cortex. Science, 307, 1118-1121.

Brown, J. W., Bullock, D., \& Grossberg, S. (2004). How laminar frontal cortex and basal ganglia circuits interact to control planned and reactive saccades. Neural Networks, 17, 471-510.

Cohen, M. X., \& Ranganath, C. (2007). Reinforcement learning signals predict future decisions. Journal of Neuroscience, 27, 371-378.

Curran, T., DeBuse, C., \& Leynes, P. A. (2007). Conflict and criterion setting in recognition memory. Journal of Experimental Psychology: Learning, Memory, \& Cognition, 33, 2-17.

Davies, P. L., Segalowitz, S. J., Dywan, J., \& Pailing, P. E. (2001). Error-negativity and positivity as they relate to other ERP indices of attentional control and stimulus processing. Biological Psychology, 56, 191-206.

Debener, S., Ullsperger, M., Siegel, M., Fiehler, K., von CraMON, D. Y., \& ENGEL, A. K. (2005). Trial-by-trial coupling of concurrent electroencephalogram and functional magnetic resonance imaging identifies the dynamics of performance monitoring. Journal Neuroscience, 25, 11730-11737.

DIEN, J. (1998). Issues in the application of the average reference: Review, critiques, and recommendations. Behavior Research Methods, Instruments, \& Computers, 30, 34-43.

Eblen, F., \& Graybiel, A. M. (1995). Highly restricted origin of prefrontal cortical inputs to striosomes in the macaque monkey. Journal of Neuroscience, 15, 5999-6013.

Egan, M. F., Goldberg, T. E., Kolachana, B. S., Callicott, J. H., Mazzanti, C. M., Straub, R. E., Et al. (2001). Effect of COMT Val108/158 Met genotype on frontal lobe function and risk for schizophrenia. Proceedings of the National Academy of Sciences, 98 , 6917-6922.

Falkenstein, M., Hoormann, J., Christ, S., \& Hohnsbein, J. (2000). ERP components on reaction errors and their functional significance: A tutorial. Biological Psychology, 51, 87-107.

Frank, M. J. (2005). Dynamic dopamine modulation in the basal ganglia: A neurocomputational account of cognitive deficits in medicated and nonmedicated Parkinsonism. Journal of Cognitive Neuroscience, 17, 51-72.

Frank, M. J., Moustafa, A. A., Haughey, H. M., Curran, T., \& Hutchison, K. E. (2007). Genetic triple dissociation reveals multiple roles for dopamine in reinforcement learning. Proceedings of the $\mathrm{Na}$ tional Academy of Sciences, 104, 16311-16316.

Frank, M. J., \& O'ReILly, R. C. (2006). A mechanistic account of striatal dopamine function in human cognition: Psychopharmacological studies with cabergoline and haloperidol. Behavioral Neuroscience, 120, 497-517.

Frank, M. J., O'Reilly, R. C., \& Curran, T. (2006). When memory fails, intuition reigns: Midazolam enhances implicit inference in humans. Psychological Science, 17, 700-707

Frank, M. J., Santamaria, A., O'Reilly, R. C., \& Willcutt, E. (2007). Testing computational models of dopamine and noradrenaline dysfunction in attention deficit/hyperactivity disorder. Neuropsychopharmacology, 32, 1583-1599.

Frank, M. J., Seeberger, L. C., \& O'Reilly, R. C. (2004). By carrot or by stick: Cognitive reinforcement learning in Parkinsonism. Science, 306, 1940-1943.

Frank, M. J., Woroch, B. S., \& CURran, T. (2005). Error-related negativity predicts reinforcement learning and conflict biases. Neuron, 47, 495-501.

Gehring, W. J., Goss, B., Coles, M. G. H., Meyer, D. E., \& DonCHIN, E. (1993). A neural system for error detection and compensation. Psychological Science, 4, 385-390.

Gogos, J.A., Morgan, M., Luine, V., Santha, M., Ogawa, S., Pfaff, D., \& KARAYIORGOU, M. (1998). Catechol-O-methyltransferase-deficient mice exhibit sexually dimorphic changes in catecholamine levels and behavior. Proceedings of the National Academy of Sciences, 95, 9991-9996.

Gratton, G., Coles, M. G. H., \& Donchin, E. (1983). A new method for off-line removal of ocular artifact. Electroencephalography \& Clinical Neurophysiology, 55, 468-484.

HANDY, T. C. (2004). Basic principles of ERP quantification. In T. C. Handy (Ed.), Event-related potentials: A methods handbook (pp. 33-56). Cambridge, MA: MIT Press. 
Hansenne, M. (1999). P300 and personality: An investigation with the Cloninger's model. Biological Psychology, 50, 143-155.

Herrmann, M. J., Römmler, J., Ehlis, A.-C., Heidrich, A., \& FallGATTER, A. J. (2004). Source localization (LORETA) of the errorrelated-negativity (ERN/Ne) and positivity (Pe). Cognitive Brain Research, 20, 294-299.

Hewig, J., Trippe, R., Hecht, H., Coles, M. G. H., Holroyd, C. B., \& MiLTnER, W. H. R. (2007). Decision-making in blackjack: An electrophysiological analysis. Cerebral Cortex, 17, 865-877.

Hollerman, J. R., \& Schultz, W. (1998). Dopamine neurons report an error in the temporal prediction of reward during learning. Nature Neuroscience, 1, 304-309.

Holroyd, C. B., \& Coles, M. G. H. (2002). The neural basis of human error processing: Reinforcement learning, dopamine, and the errorrelated negativity. Psychological Review, 109, 679-709.

Holroyd, C. B., Praamstra, P., Plat, E., \& Coles, M. G. H. (2002). Spared error-related potentials in mild to moderate Parkinson's disease. Neuropsychologia, 40, 2116-2124.

JunghöFer, M., Elbert, T., Tucker, D. M., \& Braun, C. (1999). The polar average reference effect: A bias in estimating the head surface integral in EEG recording. Clinical Neurophysiology, 110, 1149-1155.

Kim, M.-S., Cho, S.-S., Kang, K.-W., Hwang, J.-L., \& Kwon, J. S. (2002). Electrophysiological correlates of personality dimensions measured by Temperament and Character Inventory. Psychiatry \& Clinical Neurosciences, 56, 631-635.

KuČERA, H., \& Francis, W. (1967). Computational analysis of presentday American English. Providence, RI: Brown University Press.

LEUTHOLd, H., \& SOMmer, W. (1999). ERP correlates of error processing in spatial S-R compatibility tasks. Clinical Neurophysiology, 110, 342-357.

LUCK, S. J. (2005). An introduction to the event-related potential technique. Cambridge, MA: MIT Press.

Meyer-Lindenberg, A., Straub, R. E., Lipska, B. K., Verchinski, B. A., Goldberg, T., Callicott, J. H., ET Al. (2007). Genetic evidence implicating DARPP-32 in human frontostriatal structure, function, and cognition. Journal of Clinical Investigation, 117, 672-682.

Miltner, W. H. R., Braun, C. H., \& Coles, M. G. H. (1997). Eventrelated brain potentials following incorrect feedback in a timeestimation task: Evidence for a "generic" neural system for errordetection. Journal of Cognitive Neuroscience, 9, 788-798.

Nieuwenhuis, S., Aston-Jones, G., \& Cohen, J. D. (2005). Decision making, the $\mathrm{P} 3$, and the locus coeruleus-norepinephrine system. Psychological Bulletin, 131, 510-532.

Nieuwenhuis, S., RidDerinkhof, K. R., Blom, J., Band, G. P., \& KoK, A. (2001). Error-related brain potentials are differentially related to awareness of response errors: Evidence from an antisaccade task. Psychophysiology, 38, 752-760.

Pailing, P. E., \& Segalowitz, S. J. (2004). The effects of uncertainty in error monitoring on associated ERPs. Brain \& Cognition, 56, 215-233.

Picton, T. W., Lins, O. G., \& Scherg, M. (1995). The recording and analysis of event-related potentials. In F. Boller \& J. Grafman (Eds.), Handbook of neuropsychology (Vol. 10, pp. 3-73). Amsterdam: Elsevier.
RAY, J., HANSEN, S., \& WaTERS, N. (2006). Links between temperamental dimensions and brain monoamines in the rat. Behavioral Neuroscience, 120, 85-92.

Reuter, M., \& Hennig, J. (2005). Association of the functional catechol-O-methyltransferase VAL158MET polymorphism with the personality trait of extraversion. NeuroReport, 16, 1135-1138.

Ridderinkhof, K. R., Ullsperger, M., Crone, E. A., \& NieuwenHUIS, S. (2004). The role of the medial frontal cortex in cognitive control. Science, 306, 443-446.

Sara, S. J., Dyon-Laurent, C., \& Hervé, A. (1995). Novelty seeking behavior in the rat is dependent upon the integrity of the noradrenergic system. Cognitive Brain Research, 2, 181-187.

Srinivasan, R., Nunez, P. L., Tucker, D. M., Silberstein, R. B., \& CAdusch, P. J. (1996). Spatial sampling and filtering of EEG with spline Laplacians to estimate cortical potentials. Brain Topography, 8, 355-366.

TsaI, S.-J., Hong, C.-J., Yu, Y. W.-Y., \& Chen, T.-J. (2004). Association study of catechol-O-methyltransferase gene and dopamine $\mathrm{D}_{4}$ receptor gene polymorphisms and personality traits in healthy young Chinese females. Neuropsychobiology, 50, 153-156.

Tsai, S.-J., Yu, Y. W.-Y., Chen, T.-J., Chen, J.-Y., Liou, Y.-J., Chen, M.-C., \& HonG, C.-J. (2003). Association study of a functional catechol-O-methyltransferase-gene polymorphism and cognitive function in healthy females. Neuroscience Letters, 338, 123-126.

TuCKer, D. M. (1993). Spatial sampling of head electrical fields: The geodesic sensor net. Electroencephalography \& Clinical Neurophysiology, 87, 154-163.

Tucker, D. M., Luu, P., Frishkoff, G., Quiring, J., \& Poulsen, C. (2003). Frontolimbic response to negative feedback in clinical depression. Journal of Abnormal Psychology, 112, 667-678.

Tunbridge, E. M., Bannerman, D. M., Sharp, T., \& Harrison, P. J. (2004). Catechol-O-methyltransferase inhibition improves set-shifting performance and elevates stimulated dopamine release in the rat prefrontal cortex. Journal of Neuroscience, 24, 5331-5335.

Tunbridge, E. M., Harrison, P. J., \& Weinberger, D. R. (2006). Catechol-O-methyltransferase, cognition, and psychosis: Val158met and beyond. Biological Psychiatry, 60, 141-151.

VAN VeEn, V., \& CARTER, C. S. (2002). The timing of action-monitoring processes in the anterior cingulate cortex. Journal of Cognitive Neuroscience, 14, 593-602.

Walker, A., Najarian, D., White, D., JafFe, J., Kanetsky, P., \& RebBECK, T. (1999). Collection of genomic DNA by buccal swabs for polymerase chain reaction-based biomarker assays. Environmental Health Perspectives, 107, 517-520.

Yeung, N., Botvinick, M. M., \& Cohen, J. D. (2004). The neural basis of error detection: Conflict monitoring and the error-related negativity. Psychological Review, 111, 931-959.

YeUnG, N., \& SANFeY, A. G. (2004). Independent coding of reward magnitude and valence in the human brain. Journal of Neuroscience, 24, 6258-6264.

(Manuscript received January 22, 2007; revision accepted for publication June 22, 2007.) 